OPEN ACCESS

Edited by: Jessica Lee Oliva, Monash University, Australia

Reviewed by:

Kurt Kotrschal,

University of Vienna, Austria

Sylvain Fiset,

Université de Moncton, Canada

*Correspondence:

Evan L. MacLean

evanmaclean@email.arizona.edu

Specialty section:

This article was submitted to Comparative Psychology, a section of the journal Frontiers in Psychology

Received: 25 March 2017 Accepted: 01 September 2017 Published: 27 September 2017

Citation:

MacLean EL, Gesquiere LR, Gruen ME, Sherman BL, Martin WL and Carter CS (2017) Endogenous

Oxytocin, Vasopressin, and Aggression in Domestic Dogs.

Front. Psychol. 8:1613

doi: 10.3389/fpsyg.2017.01613

\section{Endogenous Oxytocin, Vasopressin, and Aggression in Domestic Dogs}

\author{
Evan L. MacLean ${ }^{1 *}$, Laurence R. Gesquiere ${ }^{2}$, Margaret E. Gruen ${ }^{3}$, Barbara L. Sherman ${ }^{4}$, \\ W. Lance Martin ${ }^{5}$ and C. Sue Carter ${ }^{6}$ \\ ${ }^{1}$ School of Anthropology, University of Arizona, Tucson, AZ, United States, ${ }^{2}$ Department of Biology, Duke University, Durham, \\ NC, United States, ${ }^{3}$ Evolutionary Anthropology, Duke University, Durham, NC, United States, ${ }^{4}$ Department of Clinical \\ Sciences, NC State College of Veterinary Medicine, NC State University, Raleigh, NC, United States, ${ }^{5}$ Martin-Protean LLC, \\ Princeton, NJ, United States, ${ }^{6}$ Kinsey Institute and Department of Biology, Indiana University, Bloomington, IA, United States
}

Aggressive behavior in dogs poses public health and animal welfare concerns, however the biological mechanisms regulating dog aggression are not well understood. We investigated the relationships between endogenous plasma oxytocin (OT) and vasopressin (AVP) - neuropeptides that have been linked to affiliative and aggressive behavior in other mammalian species-and aggression in domestic dogs. We first validated enzyme-linked immunosorbent assays (ELISAs) for the measurement of free (unbound) and total (free + bound) OT and AVP in dog plasma. In Experiment 1 we evaluated behavioral and neuroendocrine differences between a population of pet dogs with a history of chronic aggression toward conspecifics and a matched control group. Dogs with a history of aggression exhibited more aggressive behavior during simulated encounters with conspecifics, and had lower free, but higher total plasma AVP than matched controls, but there were no group differences for OT. In Experiment 2 we compared OT and AVP concentrations between pet dogs and a population of assistance dogs that have been bred for affiliative and non-aggressive temperaments, and investigated neuroendocrine predictors of individual differences in social behavior within the assistance dog population. Compared to pet dogs, assistance dogs had higher free and total OT, but there were no differences in either measure for AVP. Within the assistance dog population, dogs who behaved more aggressively toward a threatening stranger had higher total AVP than dogs who did not. Collectively these data suggest that endogenous OT and AVP may play critical roles in shaping dog social behavior, including aspects of both affiliation and aggression.

Keywords: oxytocin, vasopressin, aggression, dog, behavior

\section{INTRODUCTION}

Aggressive behavior in dogs is a serious concern for reasons related to both public health and animal welfare. In the United States it is estimated that dogs bite $\sim 4.5$ million Americans annually, with approximately half of these bites directed toward children (Centers for Disease Control and Prevention, 2003; Gilchrist et al., 2008). In addition to this impact on human lives, aggression (toward humans or other dogs) is also one of the most common reasons that dogs are relinquished to animal shelters (Salman et al., 1998), with $\sim 2$ million of these dogs being euthanized every year (Patronek et al., 1996). Despite these widely recognized concerns, we know relatively little about the psychological and biological factors underlying dog aggression. 
Aggression is an adaptive-but occasionally pathologicalform of social behavior which can be motivated by a wide range of psychological states. Dog aggression may result from cognitive appraisals of the social environment which result in fear, anger, or, predatory motivations, and individuals may differ in their tendency to express or inhibit behavioral responses to these emotions. The biological mechanisms which facilitate aggressive behavior are diverse, and different forms of aggression may be mediated by different neurobiological substrates (Panksepp and Zellner, 2004).

Previous studies on the biology of canine aggression have focused predominantly on the role of androgens and the serotonergic system. Although, testosterone is positively associated with aggression in many species (Archer, 1988), studies of androgens and aggression in dogs have been largely inconclusive. For example, in some studies gonadectomy (which yields decreased androgen production) has been linked to a mild reduction in male dog aggression toward both other dogs and people (Neilson et al., 1997), whereas in others neutered dogs were found to be more aggressive (Guy et al., 2001). Findings on the serotonergic system have been more consistent than those for androgens. Specifically, some dogs with a history of aggression are characterized by low levels of serotonin or serotonin metabolites-in both blood and cerebrospinal fluid (CSF) - and this finding is especially pronounced in lineages prone to aggression (Reisner et al., 1996; Haug, 2008; Rosado et al., 2010; León et al., 2012; Amat et al., 2013). Because of the inhibitory effect of serotonin on aggression, one common intervention for aggressive dogs has been to increase serotonin availability through selective serotonin reuptake inhibitors (SSRIs; Haug, 2008). Although, testosterone and serotonin may both have important roles in regulating aggression, research with other mammalian species indicates that oxytocin and arginine vasopressin also play major roles in the inhibition and facilitation of aggressive behaviors (Carter, 1998; Caldwell et al., 2008; Albers, 2012). However, few studies have investigated the links between these neuropeptides and aggressive behavior in dogs.

Oxytocin (OT) and arginine vasopressin (AVP) are closely related nonapeptides with wide ranging effects on social behavior, cognition, and stress responses (Carter, 1998; Goodson and Bass, 2001; Carter et al., 2008; Donaldson and Young, 2008). Although, the biological effects of OT and AVP can be similar in many cases, in others they are antagonistic (Neumann and Landgraf, 2012). With respect to affective states and social behavior, OT inhibits the sympathoadrenal axis, reduces anxiety, and can promote affiliative behavior. In contrast, AVP increases sympathoadrenal activity, is anxiogenic, and in some cases facilitates aggression (Ferris, 1992; Carter, 1998). However, both peptides can have effects that are sex- and species-specific, and depend on site of action in the brain, as well as characteristics of the receptor (Kelly and Goodson, 2014). Moreover, both peptides are capable of binding to one another's receptors, and the dynamic balance between OT and AVP is hypothesized to mediate a wide spectrum of emotional states and social behaviors (Neumann and Landgraf, 2012).

Recent studies with dogs have highlighted the role of OT in affiliative behavior and positive affective states. For example, dogs exhibit an increase in OT after friendly interaction with a human (Odendaal and Meintjes, 2003; Rehn et al., 2014; Nagasawa et al., 2015; MacLean et al., 2017b), or other pleasurable experiences (Mitsui et al., 2011; Beetz et al., 2012). Recently, polymorphisms in the oxytocin receptor gene (OXTR) have been linked to human-directed social behavior in dogs (Kis et al., 2014; Oliva et al., 2016b), and dogs treated with intranasal OT have been documented to exhibit increased affiliative behavior toward both humans and other dogs (Romero et al., 2014; Nagasawa et al., 2015; but see Hernádi et al., 2015). Lastly, OT administration has been documented to enhance some aspects of dog-human communication (Oliva et al., 2015), including cognitive skills that may be convergent between humans and dogs (MacLean and Hare, 2015; MacLean et al., 2017a). Thus, current data suggest that OT both facilitates and responds to some types of affiliative and cooperative social interaction in dogs.

Although, no studies have investigated the role of AVP in dog aggression, data from other mammalian species suggest that AVP plays an important role in regulating aggression toward unfamiliar individuals. For example, early studies on AVP and aggression revealed that microinjection of AVP into the hypothalamus of golden hamsters led to increased aggression toward unfamiliar conspecifics, whereas hamsters receiving an AVP antagonist displayed a dose-dependent decrease in biting and latency to attack unfamiliar individuals (Ferris and Potegal, 1988; Ferris, 1992; Ferris et al., 1997, 2006; Albers, 2012). Although, these findings have been replicated in several other species (Bester-Meredith et al., 2005; Gobrogge et al., 2007), other experiments reveal that AVP can both facilitate or inhibit aggression, depending on the site of action in the brain or sexspecific factors (Kelly and Goodson, 2014; reviewed in Albers, 2015), and AVP may be critical for some forms of affiliative behavior (Carter et al., 1995). In contrast to these rodent studies which have addressed localized functions of AVP, human studies have measured AVP in cerebrospinal fluid (CSF) or the periphery to assess potential links between overall circulating levels of AVP and social behavior. With respect to aggression, Coccaro et al. (1998) measured AVP in human CSF and found positive associations between AVP and a life history of aggression, and studies administering intranasal AVP in men led to decreased perceptions of friendliness in unfamiliar faces (Thompson et al., 2006).

Taken together, these findings suggest that OT may play a larger role in affiliative social behavior, anxiolysis, and the inhibition of aggression, whereas AVP-though also critical to bond formation and parental behavior-may play a larger role in anxiogenesis and aggression. To investigate the links between OT, AVP, and aggressive behavior in dogs we conducted two studies in which dogs were individually exposed to various stimuli: (1) three-dimensional dog models, (2) video images of other dogs, and/or (3) a threatening human, and we recorded the resulting aggressive responses. Free (unbound) and total (free + bound) plasma OT and AVP concentrations were determined and used as predictors of behavior in these contexts. We first conducted a series of methodological studies to validate sample preparation protocols for the measurement of OT and AVP in dog plasma (SOM). In Experiment 1, we compared the behavior and OT/AVP 
concentrations of two group of dogs: a "case group"-dogs recruited because of their known history of aggression toward unfamiliar conspecifics - and a "control group" - dogs with no previous history of aggression toward conspecifics, who were matched to cases on the basis of breed, sex, and age. These dogs were exposed to life-like three-dimensional dog models, as well as video-projected stimuli featuring dogs engaged in a variety of non-aggressive behaviors. In Experiment 2 we compared the hormone concentrations of a population of assistance dogswho have been selectively bred for affiliative and non-aggressive behavior-and the companion dogs tested in Experiment 1. We also tested the assistance dogs with the video stimuli used in Experiment 1, as well as in a temperament evaluation during which dogs were exposed to a life-like three-dimensional dog model, and an unfamiliar human who approached the dog in a threatening manner.

\section{GENERAL METHODS FOR EXPERIMENTS 1-2}

Oxytocin samples were assayed using commercially available enzyme-linked immunosorbent assay (ELISA) kits from Arbor Assays (K048) and Cayman Chemical (500440). The Arbor Assays kit was used for all analyses with the exception of the measurement of free OT with the assistance dog population in Experiment 2. This change was implemented because free OT concentrations measured with the Arbor Assays kit in Experiment 1 were near the lower limit of detection, and subsequent analyses in our lab revealed that free OT in dog plasma was detectable in a better region of the standard curve with the Cayman Chemical kit. All vasopressin samples were assayed using a commercially available ELISA kit from Enzo Life Sciences (ADI-900-017A).

Recent data suggest that OT binds strongly to plasma proteins which may prevent its detection in plasma (Martin and Carter, 2013; Martin, 2014; Brandtzaeg et al., 2016). Given its structural similarity to OT and the presence of a disulfide bridge, it is likely that AVP exhibits similar binding patterns. We have recently shown that a reduction/alkylation and protein precipitation (R/A PPT) procedure-which liberates bound OT from plasma proteins-allows for the detection of much higher concentrations of OT, and have validated this approach with dog plasma analyzed by ELISA (Brandtzaeg et al., 2016). Due to the protein precipitation step, this process also eliminates the matrix interferences commonly observed when working with neat plasma. Here we used this approach for both OT and AVP (see SOM).

For the measurement of free OT and AVP, all samples were processed using solid phase extraction (SPE) as described in the Supplemental Materials (SOM). We expected that samples processed using solid phase extraction (SPE) should capture "free" peptide concentrations, reflecting acute activity at the time of the study, whereas samples prepared via R/A PPT should represent total OT and AVP concentrations, and provide a biomarker of longer-term individual differences (free and bound concentrations; Brandtzaeg et al., 2016).

\section{EXPERIMENT 1}

Experiment 1 was a case-control study in which dogs with a history of aggression (hereafter cases) toward unfamiliar dogs while walking on leash ("leash aggression") were compared to a matched control group (hereafter "controls") with no history of aggression. We opted to study leash aggression specifically, because we aimed to investigate aggressive behavior in a highly controlled context, in which all dogs could be kept a fixed distance from a controlled stimulus used to elicit aggressive responses. All testing took place at the Veterinary Health and Wellness Center at North Carolina State University's College of Veterinary Medicine.

\section{Method \\ Subjects}

Cases were recruited through dog-related email list-serves and area dog trainers specializing in cases of leash aggression. Recruitment materials solicited owners with dogs who routinely snarl, growl, or lunge at unfamiliar dogs while on leash. Individuals expressing an interest in the study participated in an initial phone screening to verify that their dog exhibited a chronic pattern of aggression toward other unfamiliar dogs, and met the inclusionary criteria described below. When cases met the inclusionary criteria, matched controls (based on breed, sex, and age) were recruited from a database of pet owners maintained by the Duke Canine Cognition Center. Owners of control dogs participated in an initial screening to verify that their dog was not aggressive toward unfamiliar conspecifics, and that dogs met the basic inclusionary criteria. In some cases, dog owners canceled their appointments after one member of a case-control dyad had been tested or scheduled, in which case we retained data from the unmatched subject and assigned this dog to the nearest case-control grouping based on sex, age, and breed. In cases when an exact breed match was not possible, we attempted to match subjects based on breed group.

All owners were offered a free veterinary exam, complete blood count (CBC), and serum biochemistry panel for their dog in exchange for participation. To be eligible for participation subjects were required to be between 1 and 9 years of age, 4.5$70 \mathrm{~kg}$ in weight, spayed, or neutered, and to have an up-todate rabies vaccination. Dogs were excluded from participation if they had chronic illnesses, a history of aggression toward humans or familiar dogs within the household, abnormal results for the $\mathrm{CBC}$ or chemistry panel, or had received psychoactive medications within the past 30 days. In total, 46 dogs began the study, but three subjects were subsequently excluded either because blood could not be collected during the veterinary exam $(N=2)$, or due to abnormalities in the $\mathrm{CBC}$ results $(N=1)$. Subject demographics for matched cases and controls are shown in Table 1. All dog owners signed informedconsent documents prior to participation and testing procedures adhered to regulations set forth by the Institutional Animal Care and Use Committee at North Carolina State University (Protocol\#: 14-184-O). 
TABLE 1 | Subject demographics for Experiment 1.

\begin{tabular}{|c|c|c|c|c|}
\hline Breed & Group & Case/Control & Sex & $\begin{array}{l}\text { Age } \\
\text { (years) }\end{array}$ \\
\hline German shepherd & A & Case & $\mathrm{F}$ & 6 \\
\hline German shepherd & A & Control & $\mathrm{F}$ & 9 \\
\hline Beagle mix & $\mathrm{B}$ & Case & $\mathrm{F}$ & 7 \\
\hline Beagle & $\mathrm{B}$ & Control & $\mathrm{F}$ & 4 \\
\hline American staffordshire terrier mix & C & Case & $\mathrm{M}$ & 4.5 \\
\hline American staffordshire terrier mix & C & Control & M & 4 \\
\hline Weimaraner & $\mathrm{D}$ & Case & M & 6 \\
\hline Golden retriever & $\mathrm{D}$ & Control & $\mathrm{M}$ & 7 \\
\hline Golden retriever & $E$ & Case & $\mathrm{F}$ & 3 \\
\hline Golden retriever & $E$ & Control & $\mathrm{F}$ & 4 \\
\hline Potcake dog & $\mathrm{F}$ & Case & $\mathrm{M}$ & 2 \\
\hline Labradoodle & $\mathrm{F}$ & Control & M & 4 \\
\hline American staffordshire terrier & G & Case & $\mathrm{F}$ & 2 \\
\hline American staffordshire terrier mix & G & Control & $\mathrm{F}$ & 3 \\
\hline Lhasa apso mix & $\mathrm{H}$ & Case & $\mathrm{M}$ & 8 \\
\hline Cavalier king charles spaniel & $\mathrm{H}$ & Control & $\mathrm{M}$ & 7 \\
\hline German shepherd & 1 & Case & $\mathrm{M}$ & 4 \\
\hline German shepherd & 1 & Control & $\mathrm{M}$ & 4 \\
\hline Hound mix & J & Case & M & 6 \\
\hline American staffordshire terrier mix & $J$ & Case & $\mathrm{M}$ & 5 \\
\hline Treeing walker coonhound & J & Control & $\mathrm{M}$ & 6 \\
\hline Corgi mix & K & Case & $\mathrm{F}$ & 3 \\
\hline Corgi mix & K & Control & $\mathrm{F}$ & 6 \\
\hline Australian cattle dog mix & $\mathrm{L}$ & Case & $\mathrm{F}$ & 3 \\
\hline Border collie mix & $\mathrm{L}$ & Control & $\mathrm{F}$ & 3 \\
\hline Boxer & $\mathrm{M}$ & Case & $\mathrm{M}$ & 5 \\
\hline Boxer & M & Control & M & 3 \\
\hline Poodle (standard) & $N$ & Case & $\mathrm{M}$ & 5 \\
\hline Mastiff mix & $\mathrm{N}$ & Case & $\mathrm{M}$ & 4.5 \\
\hline Poodle (standard) & $\mathrm{N}$ & Control & $\mathrm{M}$ & 6 \\
\hline Terrier mix & $\mathrm{O}$ & Case & $\mathrm{M}$ & 6 \\
\hline Border collie mix & $\mathrm{O}$ & Control & $\mathrm{M}$ & 6 \\
\hline Golden retriever & $\mathrm{P}$ & Case & $\mathrm{F}$ & 4 \\
\hline Golden retriever & $\mathrm{P}$ & Control & $\mathrm{F}$ & 3 \\
\hline Greyhound & $Q$ & Case & $\mathrm{M}$ & 4 \\
\hline Greyhound & $Q$ & Control & $\mathrm{M}$ & 2.5 \\
\hline Labrador retriever & $\mathrm{R}$ & Case & $\mathrm{M}$ & 8 \\
\hline Labrador retriever & $\mathrm{R}$ & Control & $\mathrm{M}$ & 9 \\
\hline Australian cattle dog & S & Case & $\mathrm{M}$ & 5 \\
\hline Australian cattle dog & S & Control & $\mathrm{M}$ & 8 \\
\hline Australian shepherd mix & $\mathrm{T}$ & Case & $\mathrm{M}$ & 7 \\
\hline Australian cattle dog & $\mathrm{T}$ & Control & $\mathrm{M}$ & 4 \\
\hline
\end{tabular}

\section{Apparatus and Stimuli}

A schematic of the testing room is shown in Figure 1. The test room was divided into two sections by a wall of filing cabinets $(1.22 \mathrm{~m}$ high) with a $1.7 \mathrm{~m}$ gap in this wall to allow subjects to view stimuli presented in the other half of the room. An opaque curtain was hung across this gap so that subjects could see through to the other side of the room only during periods of stimulus presentation. Owners sat in a chair $2.5 \mathrm{~m}$ from the dividing wall with a rubber mat adjacent to this chair where dogs were positioned at the start of the study. All dogs wore a $1.25 \mathrm{~m}$ leash attached to a neck collar to allow them to move freely within a fixed radius from their starting position during the test. Video

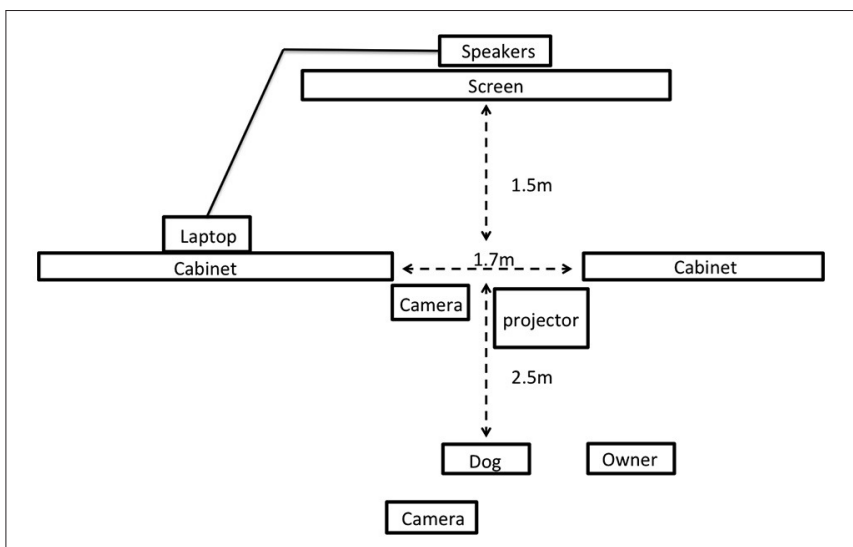

FIGURE 1 | Schematic of the testing room for Experiment 2 (not to scale).

stimuli were projected with an NEC video projector (model VT695; only present during video trials) on a $1 \times 1.5 \mathrm{~m}$ white poster board that was positioned $1.5 \mathrm{~m}$ behind the opening in the room divider. Audio speakers were positioned behind this poster board so that sounds were presented from directly behind the area where visual stimuli were presented. All trials were filmed with two high definition camcorders, one positioned at the back of the room, which captured the stimulus presentations and dog (from the rear), and the other from the gap in the center of the room, which captured the dog's behavior (oriented toward the dog's face). This second camera was the primary angle used for all behavioral coding.

Three-dimensional stimuli consisted of life-like dog models of three different sizes (small-Jack Russell Terrier; mediumShetland Sheepdog; large-Old English Sheepdog) and three inanimate objects (control stimuli) of comparable size (yellow box, black trash bag filled with paper, inflated blue yoga ball). Prior to the presentation of each stimulus we played a brief $(\sim 2 \mathrm{~s})$ auditory stimulus to attract the dog's attention to the area in which the visual stimulus would be presented. For dog models these stimuli were barking sounds appropriate for the specific model's body size, and for control stimuli these sounds were arbitrary sound effects. Video stimuli (each $15 \mathrm{~s}$ long) consisted of footage from DOGTV (a company which produces digital content optimized for dog vision). Dog clips included images of a dog being walked on leash, a dog resting in the grass, and two dogs playing. Control clips included images of water running across rocks, and panoramas of a forest canopy or flowers. Audio content was added to these clips such that all control clips had soft instrumental music, and dog clips included the sound of the dog panting, or of dog play vocalizations. The audio for dog clips was digitally edited so that the sounds and visual images were synchronous.

\section{Procedure}

\section{Veterinary Exam and Background Information}

After scheduling their research visit, all participants were provided with an electronic link to complete the Canine Behavioral and Research Questionnaire (C-BARQ), a validated and reliable instrument that measures various aspects of canine 
behavior, and has been used in previous studies of canine aggression (Duffy et al., 2008; van den Berg et al., 2010). Upon arrival, clients were escorted to a quiet consultation room where a veterinary technician collected background information about the dog's behavioral and medical history, and clients signed informed consent documentation. Dogs were then taken to a nearby exam room and the attending veterinarian performed a physical examination and collected the first blood sample to be used for a CBC, chemistry panel, and analysis of free and total OT and AVP. At the conclusion of the exam, dogs and their owners were brought to the test room where the primary experimenter (E1) explained the procedure and provided instructions for handling the dog during the test. Owners were asked to hold the dog's leash against a fixed point on the arm of their chair, and to refrain from interacting with their dog during the test trials, regardless of the dog's behavior.

\section{Three-Dimensional Stimuli}

At the start of each trial, the curtain was closed preventing dogs from viewing activity on the experimenter's side of the room. E1 positioned himself behind the curtain with the stimulus, and a second experimenter (E2) played the audio clip to attract the dog's attention. E2 then opened the curtain with a pulley, and dogs observed E1 and the stimulus for $15 \mathrm{~s}$ at which point the curtain was closed. For dog stimuli, E1 held a leash that was looped around the model dog's neck, and gently petted the dog. For control stimuli, E1 performed comparable motions (e.g., touching or patting the box and bag, or rotating and lightly bouncing the ball). E1 looked at the stimulus throughout the trial and avoided eye contact with the subject. Six trials were conducted and the stimulus type (dog model, control object) alternated between trials. We used two fixed orders for the stimulus presentation (order 2 was the reverse of order 1) and the stimulus order was counterbalanced within groups (case and control) and consistent within matched case-control pairs. Examples of the procedure and subject responses are shown in Movie S1.

\section{Video Stimuli}

At the conclusion of the final three-dimensional stimulus the curtain was left open, E1 left the testing area and started the video. Like the three-dimensional stimuli, the order of video stimuli alternated between clips featuring dogs, and control clips (nature scenes), and we used two orders of stimuli presentation that were counterbalanced as described above. Each clip was separated by $10 \mathrm{~s}$ during which the screen was black. At the conclusion of the final video stimulus dogs were taken to a nearby exam room and a second blood sample was collected to assess changes in free OT and AVP after exposure to the test stimuli.

\section{Scoring and Analysis}

From video we coded each trial for the duration of barking and growling, as well as the number of times that the dog lunged at the stimulus, or raised her upper lip in an aggressive display. Raised hackles (erectile hairs along the back of the dog) could not be coded due to heterogeneity in coat type in this diverse sample, which precluded comparable measures across
TABLE 2 | Behavioral differences between the test (dog) and control (non-dog) conditions with three-dimensional and video-projected stimuli.

\begin{tabular}{llcc}
\hline & Behavior & $\boldsymbol{t}$ & $\boldsymbol{p}$ \\
\hline 3D stimuli & Bark & -2.42 & 0.02 \\
& Growl & -2.38 & 0.02 \\
& Lunge & -3.08 & $<0.01$ \\
\hline \multirow{2}{*}{ Video stimuli } & Bark & -1.03 & 0.31 \\
& Growl & -1.51 & 0.14 \\
& Lunge & - & - \\
\hline
\end{tabular}

Dogs behaved more aggressively toward the conspecific models when presented with three-dimensional, but not video stimuli (video stimuli were not sufficient to invoke aggressive responses). Lunging was not observed in response to the video.

subjects. All trials were coded by two independent observers blind to the hypotheses and inter-rater agreement was excellent for all measures (bark: $R=0.95$; growl: $R=0.98$; lunge: $R=$ 0.95 , raise lip: $R=0.89$ ). Preliminary analyses revealed much stronger reactions to the three dimensional stimuli than the video stimuli, with very few dogs exhibiting aggressive responses to the latter (Table 2). Therefore, our analyses of behavior during the test were restricted to trials incorporating the three-dimensional stimuli. Prior to analysis, each subjects' scores were averaged across the three test trials involving dog stimuli (i.e., excluding trials with control objects) and all data were standardized by conversion to z-scores (within each behavioral category). Because a raised-lip display was observed in only one dog, this variable was dropped from analysis. For the purpose of generating a composite index of aggressive behavior, we conducted a principal components analysis with the $\mathrm{z}$-scores for barking, growling and lunging. The first principal component was loaded positively by all three variables, accounted for $54 \%$ of variance, and scores for this component were used as the primary measure of aggression (hereafter "composite aggression score").

Hormonal predictors of case-control status were assessed using conditional logistic regression (Gail et al., 1981). Unless otherwise noted, comparisons of behavior between the case and control groups were implemented using linear or generalized linear mixed models with the pair identifier for each matchedpair as a random effect. For hormonal analyses we included sex, age, body mass, and assay plate as covariates, and for behavioral analyses we included sex, age, and body mass as covariates. In all cases, individual model predictors were evaluated in a modelcomparison framework using a likelihood ratio test to determine the change in likelihood when individual variables were added to the model. Hormone data were $\log$ transformed to better meet the assumptions of parametric analysis, and subsequently converted to $z$-scores to facilitate the interpretation of regression coefficients. All analyses were conducted in the R language and environment for statistical computing (R Core Team, 2017).

\section{Sample Collection and Hormone Analysis}

All blood samples were collected into vacutainers containing K3 EDTA, centrifuged for $20 \mathrm{~min}$ at 3,000 RPMs, and the separated plasma was divided into $1 \mathrm{~mL}$ aliquots and frozen at $-80^{\circ} \mathrm{C}$ 
until assay. Samples from matched cases and controls were run on the same plate. We analyzed free OT and AVP in samples collected both before and after the experiment in order to assess short-term changes in peptide release. However, pre and post samples were highly correlated (OT: $R=0.69$; AVP: $R=0.67$ ) with no significant changes across time in either group (SOM). Therefore, we report the results only from the pre-test samples

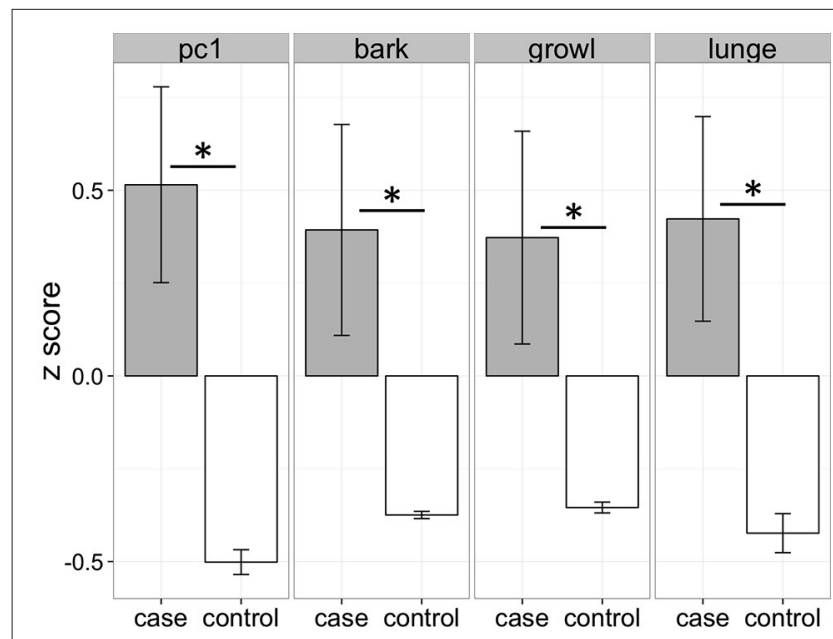

FIGURE 2 | Behavioral differences between cases and controls when confronted with lifelike three-dimensional dog models. Cases and controls differed significantly for all measures (pc1: $\chi^{2}=13.75$, df $=1, p<0.01$; bark: $\chi^{2}=8.74, \mathrm{df}=1, p<0.01$; growl: $\chi^{2}=6.27, \mathrm{df}=1, p=0.01$; lunge: $\chi^{2}=$ $7.80, \mathrm{df}=1, p<0.01)$. PC1 represents the scores from the first principal component in a principal components analysis including barking, growling, and lunging. ${ }^{*} p<0.05$. below, and additional analyses involving post-test samples, and changes across time are reported in the SOM. Total OT and AVP were measured only in pre-test samples as this measure was intended to provide a longer-term and more stable measure of individual differences.

\section{Results}

\section{Behavior}

Overall, dogs exhibited significantly more time barking and growling, and lunged at the stimulus significantly more frequently when three-dimensional dogs were presented in comparison to three-dimensional control objects (paired $t$-tests, Table 2). In contrast, video stimuli provoked very few responses in either condition, and behavior did not vary depending on whether the video featured another dog, or control content (Table 2). Therefore, analyses of behavior during the experiment focus exclusively on the trials incorporating three-dimensional stimuli.

Cases and controls differed significantly in their responses to the dog stimuli with cases exhibiting more barking, growling, and lunging than controls, as well as higher composite aggression scores (Figure 2). These behavioral differences were specific to test trials (in which dog models were presented) and the behavior of the groups did not differ during control trials (bark: $\chi^{2}=1.30$, $\mathrm{df}=1, p=0.25$; growl: $\chi^{2}=1.34, \mathrm{df}=1, p=0.25$; lunge: $\chi^{2}=$ $0.04, \mathrm{df}=1, p=0.84$ ).

\section{Free Oxytocin and Vasopressin}

A conditional logistic regression revealed that cases had significantly lower free AVP levels prior to the test than controls (Figure 3; Table 3), but that free OT concentrations did not differ
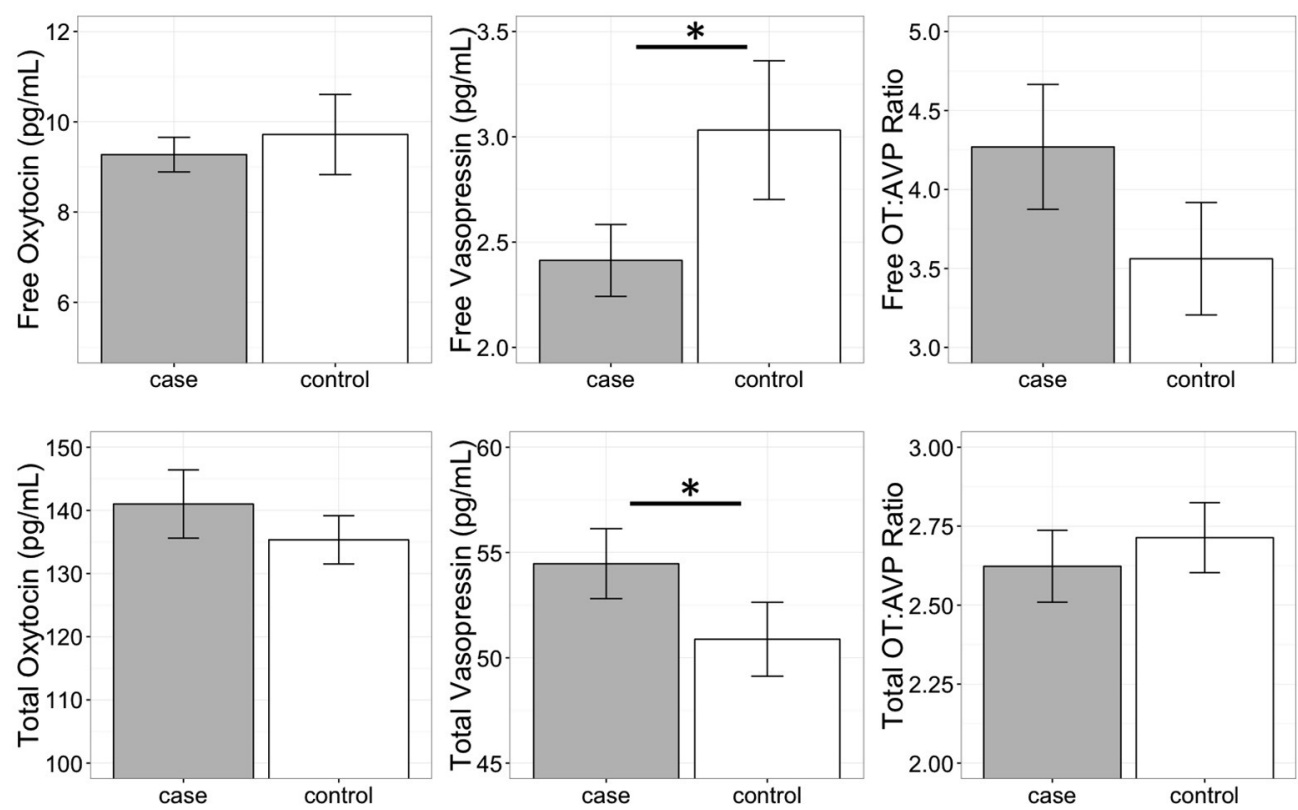

FIGURE 3 | Free and total plasma oxytocin (OT) and vasopressin (AVP) concentrations, as well as the OT:AVP ratio in dogs with a history of aggression (cases) and matched controls (no history of aggression). Cases had significantly lower free plasma AVP, and significantly higher total plasma AVP. ${ }^{\star} p<0.05$. 
TABLE 3 | Results from conditional logistic regression models in Experiment 1.

\begin{tabular}{llccccc}
\hline $\begin{array}{l}\text { Hormone } \\
\text { measure }\end{array}$ & Predictor & $\boldsymbol{\beta}$ & Odds ratio & SE $(\boldsymbol{\beta})$ & $\chi^{\mathbf{2}}$ & $\boldsymbol{p}$ \\
\hline Total & Oxytocin & 0.05 & 1.05 & 0.46 & 0.01 & 0.91 \\
& Vasopressin & 1.52 & 4.57 & 0.80 & 5.42 & $\mathbf{0 . 0 2}$ \\
& Age (years) & -0.16 & 0.85 & 0.35 & 0.21 & 0.65 \\
& Weight (kg) & -0.15 & 0.86 & 0.09 & 3.61 & 0.06 \\
\hline Free & Oxytocin & 0.04 & 1.04 & 0.57 & 0.01 & 0.94 \\
& Vasopressin & -2.50 & 0.08 & 1.08 & 10.20 & $\mathbf{0 . 0 0}$ \\
& Age (years) & -1.20 & 0.29 & 0.65 & 6.27 & $\mathbf{0 . 0 1}$ \\
& Weight (kg) & -0.22 & 0.80 & 0.11 & 5.86 & $\mathbf{0 . 0 2}$ \\
\hline
\end{tabular}

Odds ratios reflect the odds of membership in the aggressive relative to control groups (values $>1$ indicate positive associations between the predictor and aggressive group membership whereas values $<1$ indicate negative associations). Bolded values show significant associations.

between groups (Figure 3; Table 3). Specifically, a one standard deviation increase in free AVP was associated with a 0.08 odds ratio of being in the case relative to the control group. Cases tended to have higher free OT:AVP ratios, but this difference was not significant (Figure 3; Table 3). Using the C-BARQ as a measure of aggression outside the experimental context, we observed similar findings. Specifically, dogs reported to be more aggressive toward other dogs were characterized by significantly lower levels of free plasma AVP (SOM).

\section{Total Oxytocin and Vasopressin}

In contrast to the analysis of free peptide concentrations, a conditional logistic regression revealed that cases had significantly higher total AVP than controls (Figure 3; Table 3). A one standard deviation increase in AVP was associated with an odds ratio of 4.5 for being in the case relative to the control group. Neither total OT, nor the total OT:AVP ratio differed between groups (Figure 3; Table 3). Lastly, using total peptide concentrations as predictors of C-BARQ scores, total AVP was positively associated with higher levels of dog-directed aggression (SOM).

\section{Discussion}

Cases and controls differed significantly in their reactions to the three-dimensional models, with cases exhibiting higher levels of aggression than controls. Because similar behavioral differences were not observed when subjects were presented with control stimuli, this suggests that aggressive responses were both specific and social in nature, and presumably triggered by the verisimilitude of the models. However, it is noteworthy that not all cases reacted aggressively toward the dog models (SOM), despite the fact that these dogs were recruited specifically due to owner-reports of chronic aggression toward unfamiliar conspecifics. Therefore, while model dogs may provide a useful tool for research purposes, response to these inanimate models is unlikely to correlate perfectly with real-world behavior (Shabelansky et al., 2015).

AVP (but not OT) concentrations prior to the test differed significantly between cases and controls. However, the direction of this difference depended on whether only the free portion, or total peptide concentrations were considered, an issue we revisit below. Specifically, cases were characterized by lower levels of free AVP, but higher levels of total AVP. In contrast, neither OT measure differed significantly between cases and controls. This finding lends further support to the idea that while structurally and functionally related, AVP may be more strongly associated with aggression than OT.

The differences between cases and controls in this study may reflect atypical characteristics of the case population, the control population, or both. Specifically, whereas cases were recruited due to a history of aggression toward other dogs, we required that controls had not exhibited notable aggression toward conspecifics, possibly creating a contrast between dogs at extreme ends of a behavioral continuum. To address whether dogs in the control group were characterized by abnormally low levels of fear or aggression toward humans or other dogs, we compared C-BARQ scores for these dogs to the general distributions for pet dogs reported in Hsu and Serpell (2003). Average C-BARQ scores for control subjects in Experiment 1 fell within the interquartile range for large samples of pet dogs, suggesting that control subjects were not atypical with respect to these behavioral traits.

Lastly, it is important to note that although this experiment employed a case-control design, there were some limits in our ability to match subjects based on breed and age (sex was successfully matched for all groups). Specifically, in some cases where exact breed matches were not possible, we matched subjects based on breed group and morphological characteristics (e.g., body mass). Similarly, age could not be matched exactly in many cases, but on average, paired cases and controls did not differ in age by more than 1.5 years. Therefore, although we obtained reasonable groupings of cases and controls, future studies may benefit by more exact matching on these parameters.

\section{EXPERIMENT 2}

Experiment 2 evaluated hormonal predictors of variance in social behavior in a population of candidate assistance dogs tested at Canine Companions for Independence (CCI) in Santa Rosa, CA. Additionally, we compared endogenous OT and AVP levels between this population and the population of pet dogs studied in Experiment 1. Because this assistance dog population has been under active selection for friendly and non-aggressive temperaments for $>40$ years, we expected that if OT and AVP play critical roles in shaping these traits, then this population may exhibit unique neuroendocrine characteristics relative to a population of pet dogs.

\section{Method \\ Subjects}

Thirty candidate assistance dogs from CCI participated in Experiment 2. Demographic information for all subjects is shown in Table 4.

\section{Procedure}

All assistance dogs participated in an initial temperament test (designed and implemented by CCI) which included two social events (threatening stranger and unfamiliar dog) 
TABLE 4 | Subject demographics for Experiment 2.

\begin{tabular}{|c|c|c|c|}
\hline Breed & Sex & Age & Intact \\
\hline Lab-Golden cross & M & 1.8 & No \\
\hline Lab-Golden cross & $\mathrm{F}$ & 1.6 & Yes \\
\hline Lab-Golden cross & M & 1.6 & No \\
\hline Lab-Golden cross & $\mathrm{F}$ & 1.6 & Yes \\
\hline Lab-Golden cross & M & 1.6 & No \\
\hline Lab-Golden cross & $\mathrm{F}$ & 1.6 & Yes \\
\hline Lab-Golden cross & $\mathrm{F}$ & 1.6 & Yes \\
\hline Lab-Golden cross & M & 1.6 & No \\
\hline Lab-Golden cross & M & 1.6 & No \\
\hline Lab-Golden cross & $\mathrm{F}$ & 1.6 & Yes \\
\hline Lab-Golden cross & M & 1.6 & Yes \\
\hline Lab-Golden cross & $\mathrm{F}$ & 1.5 & Yes \\
\hline Lab-Golden cross & M & 1.8 & No \\
\hline Labrador retriever & M & 1.7 & Yes \\
\hline Lab-Golden cross & $\mathrm{F}$ & 1.5 & Yes \\
\hline Golden retriever & M & 1.5 & Yes \\
\hline Lab-Golden cross & $\mathrm{F}$ & 1.7 & Yes \\
\hline Golden retriever & $\mathrm{F}$ & 1.5 & Yes \\
\hline Lab-Golden cross & M & 1.7 & No \\
\hline Labrador retriever & $\mathrm{F}$ & 1.5 & Yes \\
\hline Lab-Golden cross & M & 1.7 & Yes \\
\hline Lab-Golden cross & $\mathrm{F}$ & 1.7 & Yes \\
\hline Labrador retriever & M & 1.5 & Yes \\
\hline Labrador retriever & $\mathrm{F}$ & 1.5 & Yes \\
\hline Labrador retriever & $\mathrm{F}$ & 1.5 & Yes \\
\hline Lab-Golden cross & $\mathrm{F}$ & 1.5 & Yes \\
\hline Lab-Golden cross & M & 1.7 & No \\
\hline Lab-Golden cross & $\mathrm{F}$ & 1.7 & Yes \\
\hline Lab-Golden cross & M & 1.6 & No \\
\hline Labrador retriever & $\mathrm{M}$ & 1.4 & Yes \\
\hline
\end{tabular}

which were video recorded for the purpose of this study. The larger temperament test was administered by walking dogs along a pre-determined path, which included a variety of potentially distracting, startling, or threatening stimuli. Immediately following this temperament test, dogs were also tested with the video stimuli used in Experiment 1. In addition to these behavioral tests, puppy-raisers completed C-BARQ evaluations for the majority of these subjects based on the dog's behavior at 1 year of age (the C-BARQ could not be obtained for some dogs who were raised in a prison puppy raising program). Blood draws were performed on all dogs as part of a routine veterinary examination 1 day prior to the behavioral test.

\section{Threatening Stranger (TS)}

In the threatening stranger test the handler walked the dog toward a man (TS) sitting on a bench who was wearing a hooded jacket and holding a cane. As the dog approached, the TS stood up, banged his cane on the ground, shouted toward the dog in a threatening tone, and walked $2 \mathrm{~m}$ toward the dog. During the TS's approach the handler remained stationary with the dog on leash for $\sim 10 \mathrm{~s}$ to observe the dog's reaction. The handler then encouraged the dog to approach the man by walking forward while holding the leash. Once the dog was within arm's reach of the TS, the TS removed his hood, set down his cane, kneeled, and greeted the dog in a friendly manner.

\section{Unfamiliar Dog}

In the unfamiliar dog trial subjects confronted a threedimensional life-like dog model (Old English sheepdog, identical to that from Experiment 1) as they walked (on leash, handled by a trainer) along a sidewalk. This sidewalk wrapped around the exterior of a building such that the model was first became visible at a distance of $\sim 10 \mathrm{~m}$. Trainers first led the dogs to a point $6 \mathrm{~m}$ from the model before turning around and returning to a distance of $10 \mathrm{~m}$ from the model. Trainers and dogs then made two additional approaches to distances of 3 and $0.6 \mathrm{~m}$ from the model, each time turning around and walking $\sim 5$ steps away from the model before the next approach. On fourth and final approach dogs were allowed to freely inspect the model before advancing to the next item in the temperament test.

\section{Video Stimuli}

The video stimuli and procedure were identical to Experiment 1.

\section{Scoring and Analysis}

All trials were scored from video by two independent observers.

\section{Video Stimuli}

Barking, growling, snarling, and lunging were coded as in Experiment 1. However, only barking and growling were observed, and only in a single dog, thus behavioral responses to the video stimuli were excluded from subsequent analysis.

\section{Threatening Stranger}

From video we coded whether dogs barked, growled, snarled, or lunged at the threatening stranger (TS). We also classified the dog's initial reaction to the TS into one of the following four categories: (1) Dog resisted the handler and was not easily coaxed toward the TS, (2) Dog resisted the handler but was easily coaxed toward the TS, (3) Dog moved toward TS confidently alongside the handler, (4) Dog moved toward TS confidently in front of the handler. Lastly, we used the following classifications for dogs' reactions to the TS after he removed his hood and greeted the dog (recovery): (1) Dog did not recover from threat and continued to avoid TS after his change in demeanor, (2) Dog remained skittish but was willing to greet and be touched by the TS, (3) Dog was eager to greet TS and showed no signs of fear or hesitation. Interrater agreement was excellent for all measures (bark: kappa $=1$; growl: kappa $=1$; snarl: kappa $=1$, initial reaction: $R=0.97$, recovery: $R=0.91$ ), but lunging was not observed so was dropped from analysis. For the purpose of analysis, barking, growling, and snarling were combined into a single composite aggression score. Because each variable was coded as a binary measure (1: present, 0 : absent), the composite aggression score was calculated as the sum of barking, growling and snarling for each dog (range 0-3).

\section{Unfamiliar Dog}

From video we coded whether dogs barked, growled, or snarled at the unfamiliar dog. We also classified the dog's approach toward the unfamiliar dog (UD) into one of the following four 
TABLE 5 | Results from statistical models comparing hormone concentrations between a population of pet, and candidate assistance dogs.

\begin{tabular}{llccc}
\hline Dependent measure & Predictor & $\boldsymbol{\beta}$ & $\chi^{\mathbf{2}}$ & $\boldsymbol{p}$ \\
\hline Free oxytocin & Population & -0.56 & 4.49 & $\mathbf{0 . 0 3}$ \\
& Sex & -0.37 & 2.18 & 0.14 \\
\hline Total oxytocin & Population & -0.87 & 14.39 & $<\mathbf{0 . 0 1}$ \\
& Sex & -0.03 & 0.02 & 0.89 \\
\hline Free OT/AVP ratio & Population & -1.41 & 3.78 & $\mathbf{0 . 0 5}$ \\
& Sex & 0.17 & 0.56 & 0.45 \\
\hline Free vasopressin & Population & 0.34 & 2.01 & 0.16 \\
& Sex & -0.19 & 0.63 & 0.43 \\
\hline Total vasopressin & Population & -0.30 & 1.53 & 0.22 \\
& Sex & 0.29 & 1.42 & 0.23 \\
\hline Total OT/AVP ratio & Population & -0.32 & 5.33 & $\mathbf{0 . 0 2}$ \\
& Sex & -0.10 & 0.56 & 0.45 \\
\hline
\end{tabular}

Bolded values indicate significant associations.

categories: (1) Dog resisted handler when approaching UD and was not easily coaxed toward UD (2) Dog resisted handler when approaching UD but was easily coaxed toward UD (3) Dog approached UD confidently but was easily redirected away from UD by handler (4) Dog approached UD confidently and was not easily redirected away from UD by handler. There was only one instance of barking and growling, and no instances of snarling, and these variables were therefore dropped prior to analysis. Inter-rater agreement for the approach measure was good $(R=$ $0.88)$.

\section{C-BARQ}

As in Experiment 1, we assessed the association between plasma peptide levels and C-BARQ scores for measures related to human- and dog-directed fear and aggression.

\section{Statistical Analysis}

Due to limited variability and high skew in the behavioral measures, all behavioral measures were discretized into two quantile groups corresponding to low and high scores on each measure using the Hmisc package (Harrell, 2015) in the R Environment for Statistical Computing (R Core Team, 2017). Associations with plasma peptide levels were tested by fitting generalized linear models predicting behavior as a function of OT, AVP, and sex. Individual model predictors were evaluated in a model-comparison framework using a likelihood ratio test to determine the change in likelihood when individual variables were added to the model. For population comparisons we included sex as a covariate in the analyses. Hormone data were log transformed prior to analysis to better meet the assumptions of parametric analyses, and subsequently standardized to facilitate interpretation of regression coefficients.

\section{Sample Collection and Hormone Analysis}

Samples were collected and processed as described in Experiment 1. Assistance dog samples were initially analyzed for free OT and AVP using the same methods from Experiment 1 to allow direct comparison of free OT and AVP concentrations between assistance and pet dogs. The majority of assistance dog samples $(N=19)$ were run on the same plates as pet dog samples allowing a direct comparison between these groups. Eleven assistance dog samples were run on a plate containing no pet dog samples, so to control for inter-assay variance we excluded these samples from the population comparison for free OT and AVP. For comparison within the assistance dog group, all samples were re-run on the same plate to allow the most direct comparisons within this population. For comparisons of free OT and AVP within the assistance dog population, we adopted a modified extraction protocol which yielded improved OT and AVP recovery (SOM). OT samples for this analysis were also measured using a different ELISA kit, which permitted detection in a better region of the kit's standard curve (see SOM for validation). For comparison of total OT and AVP all pet and assistance dog samples were initially run on the same plates allowing all individuals to be included in the population comparison. As above, all assistance dog samples were then rerun on the same plate allowing the most direct comparisons within this population.

\section{Results}

\section{Population Comparison}

Comparison of pet and candidate assistance dog samples revealed a population difference for both free and total OT (Table 5) but not for either measure of AVP (Table 5). Specifically, the candidate assistance dogs-who were systematically bred for calm temperaments and friendly demeanors-had higher free and total plasma OT than pet dogs (Figure 4). Additionally, assistance dogs had higher free and total OT:AVP ratios then pet dogs (Figure 4; Table 5).

\section{Threatening Stranger and Unfamiliar Dog}

Hormonal associations with behavior in the unfamiliar dog and threatening stranger test are summarized in Table 6. Although, dogs varied in their willingness to approach the unfamiliar dog and threatening stranger, there were few cases of aggressive behaviors. In the threatening stranger test, only a minority of subjects ( $\sim 20 \%)$ barked, growled, or snarled at the stranger, and there were no significant associations between these behaviors and free OT, AVP, or the OT:AVP ratio (Table 6). There were no associations between the free hormone measures and initial reactions to the threatening stranger (Table 6), however, dogs who remained skittish or were hesitant to greet the stranger after he changed demeanor had higher free OT:AVP ratios (low AVP relative to OT). Analysis of total peptide concentrations revealed that dogs who behaved aggressively toward the threatening stranger had significantly higher total AVP than dogs who did not, mirroring the patterns observed in Experiment 1 (Table 6). Dogs who behaved aggressively in this context also exhibited significantly lower OT:AVP ratios than dogs who did not (Table 6). Lastly, in the unfamiliar dog test, dogs who exhibited the most confident approaches toward the model dog were characterized by higher free OT:AVP ratios than dogs who were more reluctant to approach, and there were no other significant associations (Table 6). 

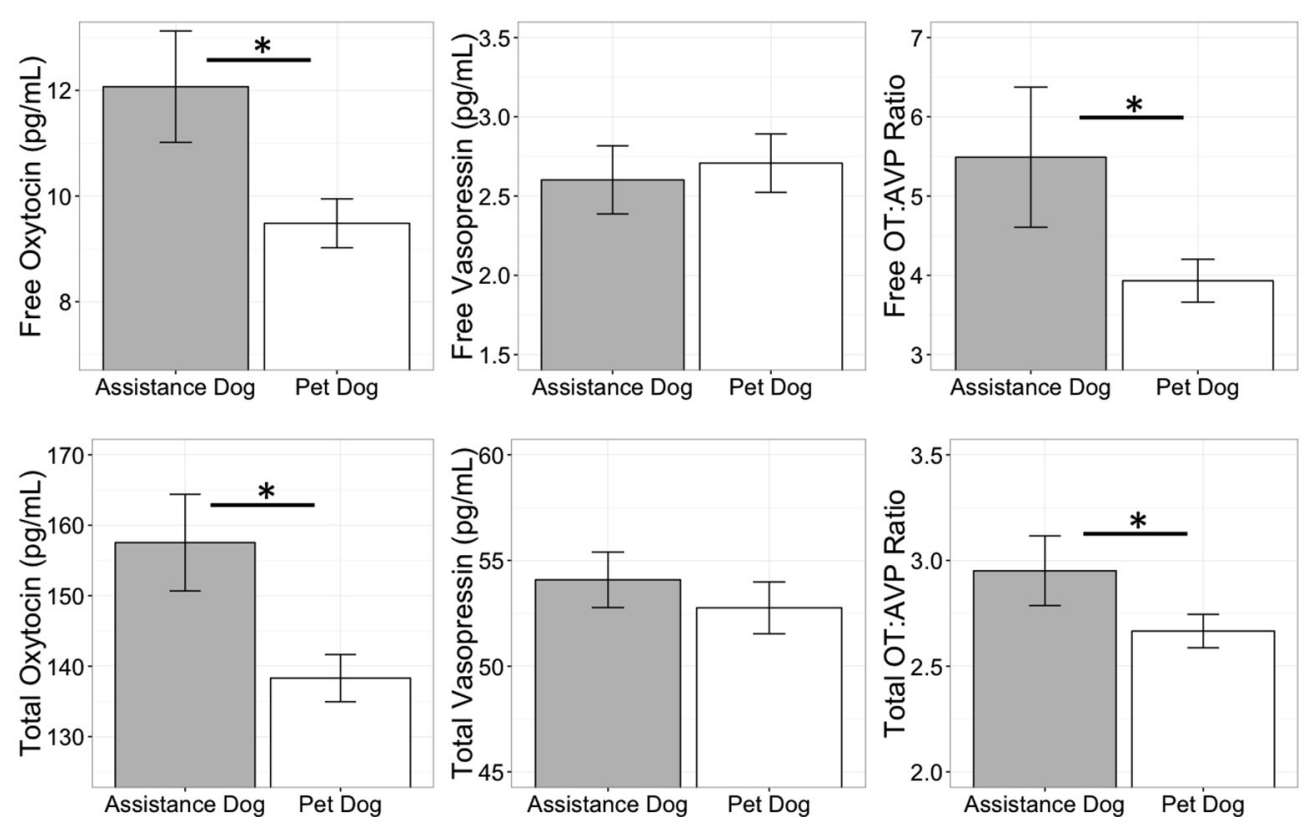

FIGURE 4 | Comparison of free and total plasma oxytocin (OT) and vasopressin (AVP) concentrations, as well as the OT:AVP ratio in pet and assistance dogs. ${ }^{*} p<0.05$.

\section{C-BARQ}

Within the assistance dog population there was minimal variation on all C-BARQ measures with the exception of dogdirected fear, which was negatively associated with the free OT:AVP ratio (SOM).

\section{Discussion}

The results of Experiment 2 are consistent with, and build on the findings from Experiment 1. Although, there was minimal aggressive behavior in this sample, dogs who behaved aggressively toward the threatening stranger had higher total AVP concentrations, and higher total AVP relative to OT, corroborating the patterns observed in Experiment 1. We also observed a population difference in OT, but not AVP concentrations between the pet dog population from Experiment 1 , and the population of candidate assistance dogs. Specifically, candidate assistance dogs had higher free and total plasma OT than pet dogs, as well as a higher ratio of OT to AVP on both measures. Given that this population has been actively selected for calm, affiliative, and non-aggressive temperaments for more than 40 years, one possibility is that this phenotype has been achieved in part due to upregulation of the oxytocinergic system. This possibility is supported by data from dog OT administration studies, in which exogenous OT has been shown to increase affiliative behavior (Romero et al., 2014; Nagasawa et al., 2015; but see Hernádi et al., 2015), and promote calm emotional states by decreasing heart rate and increasing heart-rate variability (Kovács et al., 2016). However, this population difference should be interpreted cautiously as several factors varied between the assistance and pet dog populations. Specifically, whereas the pet dog population was heterogeneous with respect to breed, age and body size, the assistance dog population consisted entirely of young retriever dogs. Given potential for breed differences in OT genetics (Bence et al., 2016) future investigations of possible differences between pet and selectively bred working populations will benefit from designs controlling for these parameters. Similarly, many individuals in the assistance dog sample were intact at the time of testing, whereas all pet dogs were sterilized. Given that OT and AVP can be affected by androgens or estrogens, it is possible that these factors may have also contributed to the observed population difference. However, within the assistance dog population, measures of both free and total OT were higher on average, among sterilized than intact dogs, making it unlikely that sterilization status accounts for the observed population difference in OT concentrations.

\section{GENERAL DISCUSSION}

Aggression is a complex and multifaceted construct that is likely to have an equally complex etiology (Blanchard et al., 2003). Aggressive behaviors may be influenced by a wide range of cognitive, genetic, epigenetic, and environmental factors, as well as an interrelated network of hormonal and physiological processes. Here we report the first systematic investigation of the relationships between endogenous oxytocin (OT), vasopressin (AVP), and aggression in domestic dogs. Our main findings were associations between plasma AVP concentrations and aggressive behavior toward both conspecifics and humans, but the direction of these relationships varied depending on whether we considered only the free fraction, or total AVP (free and bound). In Experiment 1, pet dogs with a history of chronic aggression toward conspecifics had lower free AVP, but higher total AVP than a group of controls matched on breed, sex, and age. In Experiment 2, candidate assistance dogs 


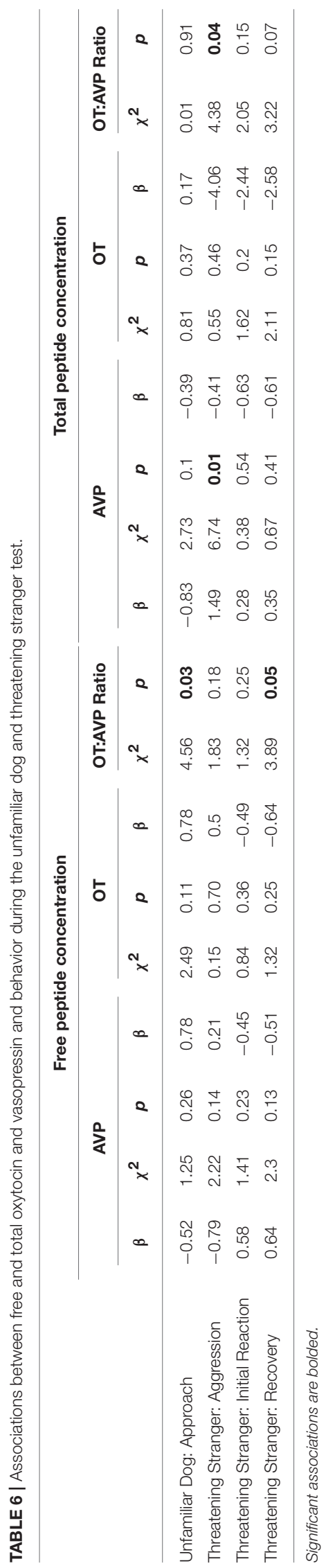

who behaved more aggressively toward a threatening (human) stranger had higher total AVP than dogs who exhibited less aggression in this context. Therefore, across studies we observed a consistent positive association between total AVP concentrations and aggressive behavior, with some evidence for an opposite association with free AVP.

As biomarkers, the significance of free vs. total peptide concentrations remains poorly understood. One possibility is that total OT/AVP may provide the most powerful approach for comparing basal individual differences, indexing long-term peptidergic activity. In contrast, free OT/AVP may be a more useful metric for assessing acute responses to stimuli. Therefore, higher total plasma AVP in dogs prone to aggression may reflect general overactivity in the vasopressinergic system, despite the fact that these dogs had lower levels of free AVP before the behavioral test. This possibility is consistent with data implicating AVP as a primary activator of the hypothalamic-pituitary-adrenal (HPA) axis (Scott and Dinan, 1998; Aguilera and RabadanDiehl, 2000), which has long been known to facilitate "fight or flight" behavior (Cannon, 1932; Kruk et al., 2004). The positive association between AVP and aggression is also consistent with previous studies in humans, which have revealed a positive correlation between AVP in cerebrospinal fluid and a life history of aggression, as well as animal studies documenting that centrally administered AVP facilitates aggression toward conspecifics, whereas AVP antagonists diminish this response (Ferris, 1992; Ferris et al., 2006).

However, a wealth of data suggests that the functions of AVP and OT may be highly dependent on species and sex-specific factors, as well as the site of action in the brain. For example, studies that have measured local AVP release, or manipulated the AVP system through receptor agonism/antagonism reveal that AVP can both facilitate and inhibit aggression (Veenema et al., 2010; Kelly and Goodson, 2014; De Boer et al., 2015). Similarly, AVP concentrations and the density of AVP tracts in various regions of the brain have also been associated with aggression, but the nature of these relationships has been variable between species (Compaan et al., 1993; Everts et al., 1997; De Boer et al., 2015).

Studies of peripheral peptide concentrations are further complicated by a lack of knowledge regarding the correspondence between central and peripheral peptide release. Although, some studies have reported positive correlations between OT and AVP concentrations in cerebrospinal fluid (CSF) and free OT/AVP in plasma, others have failed to detect this relationship (Kagerbauer et al., 2013; Carson et al., 2014, 2015). Studies of peptide release patterns have been similarly mixed with evidence for both correlated, and independent central and peripheral release (Neumann and Landgraf, 2012). Lastly, the ultimate effects of these peptides depend not only on their concentrations, but also in the distribution and state of their receptors, which vary not only between individuals and species, but also within individuals as a result of other hormonal and epigenetic processes (Carter and Porges, 2013).

Although, we found no direct associations between OT and aggression, the population of assistance dogs-which has been systematically selected for affiliative and non-aggressive 
temperaments-had higher free and total OT than the pet dog population. Given that OT has been positively associated with affiliative social behaviors, these findings are consistent with the possibility that the behavioral targets of selection in the assistance dog population are supported by OT-related mechanisms. However, the assistance and pet dog populations were not matched on critical parameters such as age, breed, or sterilization status, and future research will benefit by controlling for these factors.

At the behavioral level, these studies reveal that realistic three-dimensional models are a useful tool for evaluating dog aggression. Dogs recruited for a history of aggression toward other dogs were much more likely to behave aggressively toward these models than matched controls, and across studies, the three-dimensional models elicited stronger reactions than videoprojected stimuli. Importantly, responses to these models were not generalized reactions to novel objects, as dogs reacted much less frequently to comparably-sized control stimuli. Therefore, while unlikely to provide a perfect proxy for real-world behavior (Shabelansky et al., 2015), working with life-like models provides a highly-feasible, safe, and ethical option for assessing aggression, and may also be useful for systematic desensitization with dogs prone to aggressive behavior. In contrast, although several studies have successfully used video-projected stimuli with dogs (e.g., Pongrácz et al., 2003), our video stimuli uniformly elicited minimal response. Importantly, these video stimuli were projected to life-like dimensions, and presented with a refresh rate suitable for the flicker-fusion frequency of dog vision (Coile et al., 1989). Thus, while dogs were likely capable of perceiving the video, three-dimensional cues may be required for successfully simulating an encounter with a conspecific.

Collectively, our findings provide preliminary evidence that AVP may be an important mediator of canine aggression and call for further research in this area. Because previous studies have focused primarily on the role of serotonin and testosterone in dog aggression-both of which contribute to pathways that also include OT and AVP (Delville et al., 1996; Dölen et al., 2013)_future research will benefit by addressing the complex interactions between these systems (Weisman and Feldman, 2013). Similarly, several exploratory studies have begun to investigate the effects of intranasal peptide administration in dogs, which in some cases has led to notable changes in social behavior and cognition (Romero et al., 2014; Hernádi et al., 2015; Oliva et al., 2015, 2016a). Therefore, OT/AVP administration provides another non-invasive possibility for investigating how OT and AVP may modulate aggressive behavior in dogs. Notably, previous studies using this approach have found no evidence that intranasal OT decreases aggressive behavior in dogs (Hernádi et al., 2015), which is consistent with our findings that plasma OT levels were unrelated to individual differences in aggression.

Given that we found evidence for both positive and negative associations between AVP and aggression, we have no strong predictions regarding how AVP administration may influence aggressive behavior. Historically, AVP has been noted to have effects antagonistic to those of OT, increasing anxiety, depression, and stress responses (Benarroch, 2013). However, several studies reveal that AVP administration can have effects very similar to those of OT, decreasing heart rate (Hicks et al., 2014), reducing anxiety (Appenrodt et al., 1998), and increasing sociability (Ramos et al., 2014). Notably, many of the prosocial effects of OT, AVP, and MDMA ("Ecstasy") are believed to act through AVP networks in the brain (Ramos et al., 2013), and blocking AVP, but not OT receptors inhibits many of the effects of both OT and AVP, suggesting that the actions of both peptides are dependent on an AVP receptor-dependent mechanism (Hicks et al., 2014). Therefore, as with OT, the effects of AVP administration are likely to be complex, and moderated by a diverse range of biological factors (Bartz et al., 2011).

Ultimately, dog aggression is a normal and adaptive social behavior, but expressed in the wrong contexts, or to an extreme extent, its consequences jeopardize the welfare of both humans and dogs in our society. It is likely that dog aggression can be motivated by diverse psychological states, including fear and anger. These emotional processes may be facilitated by, or produce effects on, OT and AVP signaling in the brain. Thus, it is important to consider dog aggression at multiple levels of analysis, addressing both the cognitive processes (e.g., appraisal, learning, inhibition), and underlying physiological mechanisms, which mediate these behaviors. The studies presented here suggest that OT and AVP may play important roles in these socioemotional processes, and set the stage for future work evaluating whether treatments and interventions for aggression can be improved by considering the roles of these neuropeptides. Ultimately, we hope that these investigations will lead to increased knowledge of the biology of social behavior, promote human and animal welfare, and help to preserve the unique and long-standing relationship between humans and dogs.

\section{AUTHOR CONTRIBUTIONS}

EM, LG, MG, BS, and CC: Designed the experiments. EM, LG, MG, and BS: Conducted the experiments. EM, LG, MG, BS, WM, and CC: Wrote the article.

\section{ACKNOWLEDGMENTS}

We thank Eliot Cohen, Kerri Rodriquez, Lyndy Bleu Harden Plumley, Alexa King, Janet Bogan, Kerinne Levy, and Brenda Kennedy for help with data collection. We thank Duncan Lascelles and the Clinical Services Core at North Carolina State University College of Veterinary Medicine for their support of this research. We thank James Serpell and the University of Pennsylvania for allowing us to use the C-BARQ as a part of this study, and DOGTV for allowing us to use video footage for the purpose of these experiments. We gratefully acknowledge the Stanton Foundation for a Next Generation Canine Research Fellowship to EM, which supported this research. Grants from the Fetzer Foundation and NIH (P01 HD 075750; CC, PI) sponsored much of the preliminary work on the measurement of oxytocin which provided background for this research.

\section{SUPPLEMENTARY MATERIAL}

The Supplementary Material for this article can be found online at: http://journal.frontiersin.org/article/10.3389/fpsyg. 2017.01613/full\#supplementary-material 


\section{REFERENCES}

Aguilera, G., and Rabadan-Diehl, C. (2000). Vasopressinergic regulation of the hypothalamic-pituitary-adrenal axis: implications for stress adaptation. Regul. Pept. 96, 23-29. doi: 10.1016/S0167-0115(00)00196-8

Albers, H. E. (2012). The regulation of social recognition, social communication and aggression: vasopressin in the social behavior neural network. Horm. Behav. 61, 283-292. doi: 10.1016/j.yhbeh.2011.10.007

Albers, H. E. (2015). Species, sex and individual differences in the vasotocin/vasopressin system: relationship to neurochemical signaling in the social behavior neural network. Front. Neuroendocrinol. 36, 49-71. doi: 10.1016/j.yfrne.2014.07.001

Amat, M., Le Brech, S., Camps, T., Torrente, C., Mariotti, V. M., Ruiz, J. L., et al. (2013). Differences in serotonin serum concentration between aggressive English cocker spaniels and aggressive dogs of other breeds. J. Vet. Behav. 8, 19-25. doi: 10.1016/j.jveb.2012.04.003

Appenrodt, E., Schnabel, R., and Schwarzberg, H. (1998). Vasopressin administration modulates anxiety-related behavior in rats. Physiol. Behav. 64, 543-547. doi: 10.1016/S0031-9384(98)00119-X

Archer, J. (1988). The Behavioural Biology of Aggression, Vol. 1. CUP Archive. Cambridge: Cambridge University Press.

Bartz, J. A., Zaki, J., Bolger, N., and Ochsner, K. N. (2011). Social effects of oxytocin in humans: context and person matter. Trends Cogn. Sci. 15, 301-309. doi: 10.1016/j.tics.2011.05.002

Beetz, A., Uvnäs-Moberg, K., Julius, H., and Kotrschal, K. (2012). Psychosocial and psychophysiological effects of human-animal interactions: the possible role of oxytocin. Front. Psychol. 3:234. doi: 10.3389/fpsyg.2012.00234

Benarroch, E. E. (2013). Oxytocin and vasopressin Social neuropeptides with complex neuromodulatory functions. Neurology 80, 1521-1528. doi: 10.1212/WNL.0b013e31828cfb15

Bence, M., Marx, P., Szantai, E., Kubinyi, E., Ronai, Z., and Banlaki, Z. (2016). Lessons from the canine Oxtr gene: populations, variants and functional aspects. Genes Brain Behav. 16, 427-438. doi: 10.1111/gbb.12356

Bester-Meredith, J. K., Martin, P. A., and Marler, C. A. (2005). Manipulations of vasopressin alter aggression differently across testing conditions in monogamous and non-monogamous Peromyscus mice. Aggress. Behav. 31, 189-199. doi: 10.1002/ab.20075

Blanchard, R. J., Wall, P. M., and Blanchard, D. C. (2003). Problems in the study of rodent aggression. Horm. Behav. 44, 161-170. doi: 10.1016/S0018-506X(03)00127-2

Brandtzaeg, O. K., Johnsen, E., Roberg-Larsen, H., Seip, K. F., MacLean, E. L., Gesquiere, L. R., et al. (2016). Proteomics tools reveal startlingly high amounts of oxytocin in plasma and serum. Sci. Rep. 6:31693. doi: 10.1038/srep 31693

Caldwell, H. K., Lee, H.-J., Macbeth, A. H., and Young, I. I. I., W. S. (2008). Vasopressin: behavioral roles of an "original" neuropeptide. Progr. Neurobiol. 84, 1-24. doi: 10.1016/j.pneurobio.2007.10.007

Cannon, W. B. (1932). The Wisdom of the Body. New York, NY: W W Norton \& Co. Carson, D., Berquist, S., Trujillo, T., Garner, J., Hannah, S., Hyde, S., et al. (2015). Cerebrospinal fluid and plasma oxytocin concentrations are positively correlated and negatively predict anxiety in children. Mol. Psychiatry 20, 1085-1090. doi: 10.1038/mp.2014.132

Carson, D. S., Howerton, C. L., Garner, J. P., Hyde, S. A., Clark, C. L., Hardan, A. Y., et al. (2014). Plasma vasopressin concentrations positively predict cerebrospinal fluid vasopressin concentrations in human neonates. Peptides 61, 12-16. doi: 10.1016/j.peptides.2014.08.003

Carter, C. S. (1998). Neuroendocrine perspectives on social attachment and love. Psychoneuroendocrinology 23, 779-818. doi: 10.1016/S0306-4530(98)00055-9

Carter, C. S., Devries, A. C., and Getz, L. L. (1995). Physiological substrates of mammalian monogamy: the prairie vole model. Neurosci. Biobehav. Rev. 19, 303-314. doi: 10.1016/0149-7634(94)00070-H

Carter, C. S., Grippo, A. J., Pournajafi-Nazarloo, H., Ruscio, M. G., and Porges, S. W. (2008). "Oxytocin, vasopressin and sociality," in Progress in Brain Research, Vol. 170, eds D. N. Inga and L. Rainer (Amsterdam: Elsevier), 331-336.

Carter, C. S., and Porges, S. W. (2013). The biochemistry of love: an oxytocin hypothesis. EMBO Rep. 14, 12-16. doi: 10.1038/embor.2012.191

Centers for Disease Control and Prevention. (2003). Nonfatal Dog Bite-Related Injuries Treated in Hospital Emergency Departments-United States, 2001. MMWR: Morbidity and Mortality Weekly Report, 52, 605-610.
Coccaro, E. F., Kavoussi, R. J., Hauger, R. L., Cooper, T. B., and Ferris, C. F. (1998). Cerebrospinal fluid vasopressin levels correlates with aggression and serotonin function in personality-disordered subjects. Arch. Gen. Psychiatry 55, 708-714. doi: $10.1001 /$ archpsyc.55.8.708

Coile, D. C., Pollitz, C. H., and Smith, J. C. (1989). Behavioral determination of critical flicker fusion in dogs. Physiol. Behav. 45, 1087-1092. doi: 10.1016/0031-9384(89)90092-9

Compaan, J., Buijs, R., Pool, C., and De Ruiter, A. (1993). Differential lateral septal vasopressin innervation in aggressive and nonaggressive male mice. Brain Res. Bull. 30, 1-6. doi: 10.1016/0361-9230(93)90032-7

De Boer, S., Olivier, B., Veening, J., and Koolhaas, J. (2015). The neurobiology of offensive aggression: revealing a modular view. Physiol. Behav. 146, 111-127. doi: 10.1016/j.physbeh.2015.04.040

Delville, Y., Mansour, K. M., and Ferris, C. F. (1996). Testosterone facilitates aggression by modulating vasopressin receptors in the hypothalamus. Physiol. Behav. 60, 25-29. doi: 10.1016/0031-9384(95)02246-5

Dölen, G., Darvishzadeh, A., Huang, K. W., and Malenka, R. C. (2013). Social reward requires coordinated activity of nucleus accumbens oxytocin and serotonin. Nature 501, 179-184. doi: 10.1038/nature12518

Donaldson, Z. R., and Young, L. J. (2008). Oxytocin, vasopressin, and the neurogenetics of sociality. Science 322, 900-904. doi: 10.1126/science.1158668

Duffy, D. L., Hsu, Y., and Serpell, J. A. (2008). Breed differences in canine aggression. Appl. Anim. Behav. Sci. 114, 441-460. doi: 10.1016/j.applanim.2008.04.006

Everts, H. G., De Ruiter, A., and Koolhaas, J. M. (1997). Differential lateral septal vasopressin in wild-type rats: correlation with aggression. Horm. Behav. 31, 136-144. doi: 10.1006/hbeh.1997.1375

Ferris, C. (1992). Role of vasopressin in aggressive and dominant/subordinate behaviors. Ann. N.Y. Acad. Sci. 652, 212-226. doi: 10.1111/j.1749-6632.1992.tb34357.x

Ferris, C. F., Lu, S.-F., Messenger, T., Guillon, C. D., Heindel, N., Miller, M., et al. (2006). Orally active vasopressin V1a receptor antagonist, SRX251, selectively blocks aggressive behavior. Pharmacol. Biochem. Behav. 83, 169-174. doi: $10.1016 /$ j.pbb.2006.01.001

Ferris, C. F., Melloni, R. H. Jr, Koppel, G., Perry, K. W., Fuller, R. W., and Delville, Y. (1997). Vasopressin/serotonin interactions in the anterior hypothalamus control aggressive behavior in golden hamsters. J. Neurosci. 17, 4331-4340.

Ferris, C. F., and Potegal, M. (1988). Vasopressin receptor blockade in the anterior hypothalamus suppresses aggression in hamsters. Physiol. Behav. 44, 235-239. doi: 10.1016/0031-9384(88)90144-8

Gail, M. H., Lubin, J. H., and Rubinstein, L. V. (1981). Likelihood calculations for matched case-control studies and survival studies with tied death times. Biometrika 68, 703-707. doi: 10.1093/biomet/68.3.703

Gilchrist, J., Sacks, J., White, D., and Kresnow, M. (2008). Dog bites: still a problem? Injury Prevent. 14, 296-301. doi: 10.1136/ip.2007.016220

Gobrogge, K. L., Liu, Y., Jia, X., and Wang, Z. (2007). Anterior hypothalamic neural activation and neurochemical associations with aggression in pair-bonded male prairie voles. J. Comp. Neurol. 502, 1109-1122. doi: 10.1002/cne.21364

Goodson, J. L., and Bass, A. H. (2001). Social behavior functions and related anatomical characteristics of vasotocin/vasopressin systems in vertebrates. Brain Res. Rev. 35, 246-265. doi: 10.1016/S0165-0173(01) 00043-1

Guy, N. C., Luescher, U., Dohoo, S. E., Spangler, E., Miller, J. B., Dohoo, I. R., et al. (2001). Demographic and aggressive characteristics of dogs in a general veterinary caseload. Appl. Anim. Behav. Sci. 74, 15-28. doi: 10.1016/S0168-1591(01)00153-8

Harrell, F. E. Jr. (2015). Hmisc: Harrell Miscellaneous. R package version 3, 17-11.

Haug, L. I. (2008). Canine aggression toward unfamiliar people and dogs. Vet. Clin. N. Am. 38, 1023-1041. doi: 10.1016/j.cvsm.2008.04.005

Hernádi, A., Kis, A., Kanizsár, O., Tóth, K., Miklósi, B., and Topál, J. (2015). Intranasally administered oxytocin affects how dogs (Canis familiaris) react to the threatening approach of their owner and an unfamiliar experimenter. Behav. Process. 119, 1-5. doi: 10.1016/j.beproc.2015. 07.001

Hicks, C., Ramos, L., Reekie, T., Misagh, G., Narlawar, R., Kassiou, M., et al. (2014). Body temperature and cardiac changes induced by peripherally administered oxytocin, vasopressin and the non-peptide oxytocin receptor agonist WAY 267,464: a biotelemetry study in rats. Br. J. Pharmacol. 171, 2868-2887. doi: 10.1111/bph.12613 
Hsu, Y., and Serpell, J. A. (2003). Development and validation of a questionnaire for measuring behavior and temperament traits in pet dogs. J. Am. Vet. Med. Assoc. 223, 1293-1300. doi: 10.2460/javma.2003.223.1293

Kagerbauer, S., Martin, J., Schuster, T., Blobner, M., Kochs, E., and Landgraf, R. (2013). Plasma oxytocin and vasopressin do not predict neuropeptide concentrations in human cerebrospinal fluid. J. Neuroendocrinol. 25, 668-673. doi: $10.1111 /$ jne. 12038

Kelly, A. M., and Goodson, J. L. (2014). Social functions of individual vasopressin-oxytocin cell groups in vertebrates: what do we really know? Front. Neuroendocrinol. 35, 512-529. doi: 10.1016/j.yfrne.2014.04.005

Kis, A., Bence, M., Lakatos, G., Pergel, E., Turcsán, B., Pluijmakers, J., et al. (2014). Oxytocin receptor gene polymorphisms are associated with human directed social behavior in dogs (Canis familiaris). PLoS ONE 9:e83993. doi: 10.1371/journal.pone.0083993

Kovács, K., Kis, A., Kanizsár, O., Hernádi, A., Gácsi, M., and Topál, J. (2016). The effect of oxytocin on biological motion perception in dogs (Canis familiaris). Anim. Cogn. 19, 513-522. doi: 10.1007/s10071-015-0951-4

Kruk, M. R., Halasz, J., Meelis, W., and Haller, J. (2004). Fast positive feedback between the adrenocortical stress response and a brain mechanism involved in aggressive behavior. Behav. Neurosci. 118:1062. doi: 10.1037/0735-7044.118.5.1062

León, M., Rosado, B., García-Belenguer, S., Chacón, G., Villegas, A., and Palacio, J. (2012). Assessment of serotonin in serum, plasma, and platelets of aggressive dogs. J. Vet. Behav. 7, 348-352. doi: 10.1016/j.jveb.2012.01.005

MacLean, E. L., and Hare, B. (2015). Dogs hijack the human bonding pathway. Science 348, 280-281. doi: 10.1126/science.aab1200

MacLean, E. L., Herrmann, E., Suchindran, S., and Hare, B. (2017a). Individual differences in cooperative communicative skills are more similar between dogs and humans than chimpanzees. Anim. Behav. 126, 41-51. doi: 10.1016/j.anbehav.2017.01.005

MacLean, E. L., Gesquiere, L. R., Gee, N. R., Levy, K., Martin, W. L., and Carter, C. S. (2017b). Effects of affiliative human-animal interaction on dog salivary and plasma oxytocin and vasopressin. Front. Psychol. 8:1606. doi: 10.3389/fpsyg.2017.01606

Martin, W., and Carter, C. S. (2013). Oxytocin and Vasopressin are Sequestered in Plasma. World Congress of Neurohypophyseal Hormones Abstracts.

Martin, W. L. (2014). Measurement of Oxytocin and Vasopressin. WO Patent Application number: PCT/US2014/044,467. Google Patents. Available online at: http://google.com/patents/WO2014210399Al?cl=da

Mitsui, S., Yamamoto, M., Nagasawa, M., Mogi, K., Kikusui, T., Ohtani, N., et al. (2011). Urinary oxytocin as a noninvasive biomarker of positive emotion in dogs. Horm. Behav. 60, 239-243. doi: 10.1016/j.yhbeh.2011.05.012

Nagasawa, M., Mitsui, S., En, S., Ohtani, N., Ohta, M., Sakuma, Y., et al. (2015). Oxytocin-gaze positive loop and the coevolution of human-dog bonds. Science 348, 333-336. doi: 10.1126/science.1261022

Neilson, J. C., Eckstein, R. A., and Hart, B. (1997). Effects of castration on problem behaviors in male dogs with reference to age and duration of behavior. J. Am. Vet. Med. Assoc. 211, 180-182.

Neumann, I. D., and Landgraf, R. (2012). Balance of brain oxytocin and vasopressin: implications for anxiety, depression, and social behaviors. Trends Neurosci. 35, 649-659. doi: 10.1016/j.tins.2012.08.004

Odendaal, J., and Meintjes, R. (2003). Neurophysiological correlates of affiliative behaviour between humans and dogs. Vet. J. 165, 296-301. doi: 10.1016/S1090-0233(02)00237-X

Oliva, J., Rault, J.-L., Appleton, B., and Lill, A. (2015). Oxytocin enhances the appropriate use of human social cues by the domestic dog (Canis familiaris) in an object choice task. Anim. Cogn. 18, 767-775. doi: 10.1007/s10071-015-0843-7

Oliva, J. L., Rault, J.-L., Appleton, B., and Lill, A. (2016a). Oxytocin blocks pet $\operatorname{dog}$ (Canis familiaris) object choice task performance being predicted by owner-perceived intelligence and owner attachment. Pet Behav. Sci. 1, 31-46. doi: 10.21071/pbs.v0i1.3991

Oliva, J. L., Wong, Y. T., Rault, J.-L., Appleton, B., and Lill, A. (2016b). The oxytocin receptor gene, an integral piece of the evolution of Canis familaris from Canis lupus. Pet Behav. Sci. 2, 1-15. doi: 10.21071/pbs.v0i2. 4000

Panksepp, J., and Zellner, M. R. (2004). Towards a neurobiologically based unified theory of aggression. Rev. Int. Psychol. Soc. 17, 37-62.
Patronek, G. J., Glickman, L. T., Beck, A. M., McCabe, G. P., and Ecker, C. (1996). Risk factors for relinquishment of dogs to an animal shelter. J. Am. Vet. Med. Assoc. 209, 572-581.

Pongrácz, P., Miklósi, Á., Dóka, A., and Csányi, V. (2003). Successful application of video-projected human images for signalling to dogs. Ethology 109, 809-821. doi: 10.1046/j.0179-1613.2003.00923.x,

Ramos, L., Hicks, C., Caminer, A., and McGregor, I. S. (2014). Inhaled vasopressin increases sociability and reduces body temperature and heart rate in rats. Psychoneuroendocrinology 46, 46-51. doi: 10.1016/j.psyneuen.2014.04.013

Ramos, L., Hicks, C., Kevin, R., Caminer, A., Narlawar, R., Kassiou, M., et al. (2013). Acute prosocial effects of oxytocin and vasopressin when given alone or in combination with 3, 4-methylenedioxymethamphetamine in rats: involvement of the V1A receptor. Neuropsychopharmacology 38, 2249-2259. doi: $10.1038 / \mathrm{npp} .2013 .125$

R Core Team (2017). R: A Language and Environment for Statistical Computing. Vienna: R Foundation for Statistical Computing.

Rehn, T., Handlin, L., Uvnäs-Moberg, K., and Keeling, L. J. (2014). Dogs' endocrine and behavioural responses at reunion are affected by how the human initiates contact. Physiol. Behav. 124, 45-53. doi: 10.1016/j.physbeh.2013.10.009

Reisner, I. R., Mann, J. J., Stanley, M., Huang, Y., and Houpt, K. A. (1996). Comparison of cerebrospinal fluid monoamine metabolite levels in dominant-aggressive and non-aggressive dogs. Brain Res. 714, 57-64. doi: 10.1016/0006-8993(95)01464-0

Romero, T., Nagasawa, M., Mogi, K., Hasegawa, T., and Kikusui, T. (2014). Oxytocin promotes social bonding in dogs. Proc. Natl. Acad. Sci. U.S.A. 111, 9085-9090. doi: 10.1073/pnas.1322868111

Rosado, B., García-Belenguer, S., León, M., Chacón, G., Villegas, A., and Palacio, J. (2010). Blood concentrations of serotonin, cortisol and dehydroepiandrosterone in aggressive dogs. Appl. Anim. Behav. Sci. 123, 124-130. doi: 10.1016/j.applanim.2010.01.009

Salman, M., New, J., John, G., Scarlett, J. M., Kass, P. H., Ruch-Gallie, R., et al. (1998). Human and animal factors related to relinquishment of dogs and cats in 12 selected animal shelters in the United States. J. Appl. Anim. Welf. Sci. 1, 207-226. doi: 10.1207/s15327604jaws0103_2

Scott, L. V., and Dinan, T. G. (1998). Vasopressin and the regulation of hypothalamic-pituitary-adrenal axis function: implications for the pathophysiology of depression. Life Sci. 62, 1985-1998. doi: 10.1016/S0024-3205(98)00027-7

Shabelansky, A., Dowling-Guyer, S., Quist, H., D’Arpino, S. S., and McCobb, E. (2015). Consistency of shelter dogs' behavior toward a fake versus real stimulus dog during a behavior evaluation. Appl. Anim. Behav. Sci. 163, 158-166. doi: 10.1016/j.applanim.2014.12.001

Thompson, R., George, K., Walton, J., Orr, S., and Benson, J. (2006). Sex-specific influences of vasopressin on human social communication. Proc. Natl. Acad. Sci. U.S.A. 103, 7889-7894. doi: 10.1073/pnas.0600406103

van den Berg, S. M., Heuven, H., van den Berg, L., Duffy, D. L., and Serpell, J. A. (2010). Evaluation of the C-BARQ as a measure of stranger-directed aggression in three common dog breeds. Appl. Anim. Behav. Sci. 124, 136-141. doi: 10.1016/j.applanim.2010.02.005

Veenema, A. H., Beiderbeck, D. I., Lukas, M., and Neumann, I. D. (2010). Distinct correlations of vasopressin release within the lateral septum and the bed nucleus of the stria terminalis with the display of intermale aggression. Horm. Behav. 58, 273-281. doi: 10.1016/j.yhbeh.2010.03.006

Weisman, O., and Feldman, R. (2013). Oxytocin administration affects the production of multiple hormones. Psychoneuroendocrinology 38, 626. doi: 10.1016/j.psyneuen.2013.03.004

Conflict of Interest Statement: The authors declare that the research was conducted in the absence of any commercial or financial relationships that could be construed as a potential conflict of interest.

Copyright $\odot 2017$ MacLean, Gesquiere, Gruen, Sherman, Martin and Carter. This is an open-access article distributed under the terms of the Creative Commons Attribution License (CC BY). The use, distribution or reproduction in other forums is permitted, provided the original author(s) or licensor are credited and that the original publication in this journal is cited, in accordance with accepted academic practice. No use, distribution or reproduction is permitted which does not comply with these terms. 